\title{
PREVALÊNCIA DE HÁBITOS POSTURAIS INADEQUADOS DE ESCOLARES DO ENSINO FUNDAMENTAL DA CIDADE DE TEUTÔNIA: UM ESTUDO DE BASE POPULACIONAL'
}

\author{
MS. MATIAS NOLL \\ Instituto Federal Goiano, Campus Ceres \\ (Ceres - Goiás - Brasil) \\ Email: matiasnoll@yahoo.com.br
}

\author{
DR. CLÁUDIA TARRAGÔ CANDOTTI \\ Departamento de Educação Física e Programa de Pós-Graduação em \\ Ciências do Movimento Humano, Escola de Educação Física, \\ Universidade Federal do Rio Grande do Sul \\ (Porto Alegre - Rio Grande do Sul - Brasil) \\ Email: claudia.candotti@ufrgs.br
}

\section{DR. CARLOS LEANDRO TIGGEMANN}

Curso de Educação Física, Faculdade da Serra Gaúcha;

Curso de Educação Física, Centro Universitário UNIVATES

(Lajeado - Rio Grande do Sul - Brasil)

Email: cltiggemann@yahoo.com.br

\section{MS. MAIRA CRISTINA WOLF SCHOENELL}

Programa de Pós-Graduação em Ciências do Movimento Humano, Escola de Educação Física, Universidade Federal do Rio Grande do Sul

(Porto Alegre - Rio Grande do Sul - Brasil)

Email: mairacws@yahoo.com.br

\section{DR. ADRIANE VIEIRA}

Departamento de Educação Física e Programa de Pós-Graduação em Ciências do Movimento Humano, Escola de Educação Física,

Universidade Federal do Rio Grande do Sul

(Porto Alegre - Rio Grande do Sul - Brasil)

Email: adriane.vieira@gmail.com

I. O presente trabalho contou com financiamento da CAPES (modalidade bolsa de mestrado) e do CNPq (financiamento de projeto de pesquisa). 


\section{RESUMO}

Este estudo teve como objetivo verificar a prevalência de hábitos posturais inadequados de escolares do Ensino Fundamental da cidade de Teutônia, estado do Rio Grande do Sul, Brasil, a partir de um estudo de base populacional. Os 1597 escolares participantes responderam o questionário autoaplicável denominado Back Pain and Body Posture Evaluation Instrument (BackPEI). Os resultados demonstraram alta prevalência de hábitos posturais inadequados nas seguintes posturas: sentar para escrever e para utilizar o computador, sentar em um banco, além de pegar objeto do solo. Verificou-se também que os escolares permanecem por muito tempo na posição sentada em frente ao computador e à televisão, e não dormem a quantidade de horas necessária por dia. Estudos desta natureza podem subsidiar o trabalho preventivo dos professores de Educação Física, no ambiente escolar.

PALAVRAS-CHAVE: Postura; comportamento; saúde do adolescente; epidemiologia.

\section{INTRODUÇÃO}

Muitos dos problemas posturais que acometem a população em geral, em especial aqueles relacionados com a coluna vertebral, têm sua origem no período de crescimento e desenvolvimento corporal (PENHA et al., 2008; SMITH, O'SULLIVAN, STAKER, 2008), o qual coincide com a fase em que as crianças estão frequentando a escola. Dentre estes problemas, citam-se as algias na região das costas, as alterações de morfologia da coluna vertebral e a utilização inadequada da mecânica corporal nas atividades de vida diária (AVD's).

Considerando que os problemas relacionados à coluna vertebral possuem causa multifatorial (SIIVOLA et al. , 2004), especula-se que um dos principais fatores de risco para a ocorrência destes problemas são os hábitos posturais nas atividades do dia a dia. Hábitos posturais referem-se ao modo de utilização da mecânica corporal durante a realização das atividades de vida diária (AVD's). Quando a mecânica corporal é adequadamente utilizada, ou seja, quando as curvaturas da coluna vertebral são preservadas e a sobrecarga imposta não excede o limite fisiológico do indivíduo, entende-se que o hábito postural é adequado. Do contrário, o hábito postural do indivíduo passa a ser considerado inadequado. Por exemplo, no contexto doméstico, os hábitos posturais envolvem AVD's como pegar objetos do solo e dormir e, no contexto escolar, os hábitos posturais envolvem o modo do transporte do material escolar, a postura ao sentar para escrever e para utilizar o computador.

Dentre os hábitos posturais que fazem parte do cotidiano de escolares, o modo de transporte do material escolar tem sido amplamente investigado. Candotti, Roth e Noll (20 I 2) ao investigar escolares da cidade de Montenegro, Brasil, constataram que o meio de transporte mais utilizado foi a mochila de duas alças nas costas, e que a mesma era transportada apoiada sobre ambos os ombros, de forma 
simétrica. Resultados semelhantes foram encontrados por Aparício et al. (2005)que, ao investigarem o modo de transporte da mochila escolar em escolares da cidade de Salamanca, Espanha, verificaram que praticamente a totalidade dos avaliados utilizavam a mochila com duas alças. Entende-se que o hábito de transporte do material escolar demonstrado pelos escolares em diferentes países corresponde a um fator positivo dentre os inúmeros fatores de risco a que estão diariamente submetidos, visto que o transporte realizado de forma adequada diminui os torques de inclinação prejudiciais na coluna vertebral, que ao longo dos anos poderia resultar no surgimento de alterações posturais (WHITTFIELD; LEGG; HEDDERLY, 2005).

Não obstante, na perspectiva do estudo da postura corporal, a literatura carece de investigações, que além de investigar o modo no qual é transportado o material escolar, busquem também descrever outros hábitos relacionados aos escolares, como o hábito de sentar para escrever e utilizar o computador, hábito de dormir, entre outros, assim como o tempo de permanência em cada uma destas AVD's.

Considerando que os hábitos posturais inadequados são fatores de risco para a ocorrência problemas posturais (ROSA NETO, 1991; ANDRADE; ARAÚJO; VILAR, 2005) e que são propícios de serem desenvolvidos na fase escolar, entende-se fundamental que os mesmos sejam investigados com profundidade na infância e na juventude. Portanto, a identificação e o conhecimento dos hábitos posturais de crianças e adolescentes passam a ser relevantes para a prevenção dos problemas posturais na vida adulta, visto que estas informações podem nortear tanto o planejamento da disciplina de Educação Física, quanto da escola como um todo (NOLL et al., 20 I 2a; NOLL; CANDOTTI; VIEIRA, 20 I2). Deste modo, justifica-se a realização do presente estudo, que teve como objetivo verificar a prevalência de hábitos posturais inadequados de escolares do Ensino Fundamental da cidade de Teutônia, estado do Rio Grande do Sul, Brasil, a partir de um estudo de base populacional.

\section{MATERIAL E MÉTODOS}

Trata-se de um estudo de base populacional, realizado nos meses de agosto e setembro de $201 \mathrm{l}$. Foram convidados a participar do estudo todos os 1720 escolares do Ensino Fundamental, de $5^{\mathrm{a}}$ a $8^{\mathrm{a}}$ séries, de todas as Escolas $(\mathrm{n}=1 \mathrm{I})$ do município de Teutônia, Estado do Rio Grande do Sul, Brasil. Participaram do presente estudo 1597 escolares, sendo que apenas 7,2\% $(n=123)$ do total de escolares se recusaram a participar do presente estudo e/ou não compareceram na escola no dia da avaliação. Deste total de escolares, 835(52,3\%), 628(39,3\%) e 134(8,4\%), são de escolas da Rede Municipal, Estadual e Particular de Ensino, respectivamente. A Tabela I descreve os participantes estratificados por sexo e idade. 
Este estudo foi aprovado no Comitê de Ética e Pesquisa da Universidade Federal do Rio Grande do Sul, sob número 19832, e respeitou a Resolução 196/96 do Conselho Nacional de Saúde.

Tabela I. Frequência e percentual dos escolares avaliados estratificados por sexo e idade

\begin{tabular}{cccc}
\hline Idade & $\begin{array}{c}\text { Masculino } \\
\mathrm{N}(\%)\end{array}$ & $\begin{array}{c}\text { Feminino } \\
\mathrm{N}(\%)\end{array}$ & $\begin{array}{c}\text { Total } \\
\mathrm{N}(\%)\end{array}$ \\
\hline 11 & $99(11,6)$ & $107(14,4)$ & $206(12,9)$ \\
12 & $192(22,4)$ & $181(24,4)$ & $373(23,4)$ \\
13 & $197(23)$ & $179(24,2)$ & $376(23,5)$ \\
14 & $203(23,7)$ & $172(23,2)$ & $375(23,5)$ \\
15 & $120(14)$ & $83(11,2)$ & $203(12,7)$ \\
16 & $45(5,3)$ & $19(2,6)$ & $64(4)$ \\
Total & $856(100)$ & $74 \mid(100)$ & $1597(100)$ \\
\hline
\end{tabular}

INSTRUMENTO

Para verificar a prevalência de hábitos comportamentais e posturais foi utilizado o questionário autoaplicável denominado Back Pain and Body Posture Evaluation Instrument (BackPEI) (NOLL et al., 20 I2b). O BackPEl é um questionário válido e reprodutível, constituído por 2 I questões fechadas que possui uma versão para cada sexo (vide em Anexo). O questionário aborda questões sobre: ( I ) dor nas costas nos últimos três meses (ocorrência, frequência e intensidade); (2) demográficas (idade e sexo); (3) socioeconômicas (escolaridade dos pais/responsáveis e tipo de escola); (4) comportamentais (atividade física, ler/estudar na cama, horas/dia assistindo televisão e ao computador); (5) posturais (modo de sentar para escrever e utilizar computador, modo de transporte do material escolar, modo de dormir e modo de sentar para conversar) e (6) hereditárias (ocorrência de dor nas costas nos pais). Para o presente estudo, foram utilizadas apenas as questões 4 a 8 (referentes aos hábitos comportamentais) e as questões 9 a 14 (referentes aos hábitos posturais adotados nas AVD's).

\section{PROCEDIMENTO DE COLETA E ANÁLISE DOS DADOS}

Todas as escolas foram convidadas a participar do presente estudo por meio de uma reunião com a Secretaria Municipal de Educação (SME/Teutônia), em que foram explicados os objetivos da pesquisa, assim como os procedimentos de coleta a serem realizados. Após o consentimento da SME/Teutônia, foi agendada uma 
reunião com a direção de cada escola para apresentar o projeto de pesquisa. Uma vez dado o consentimento por todas as direções, foi agendada a data de realização das avaliações, de acordo com a agenda de cada uma das Escolas de Ensino Fundamental de $5^{\mathrm{a}}$ a $8^{\mathrm{a}}$ séries de Teutônia. $\bigcirc$ pesquisador responsável pela aplicação dos questionários avaliou cada turma individualmente, em sua própria sala de aula. Inicialmente, o pesquisador explicou coletivamente como deveria ser preenchido o questionário, o qual a seguir foi distribuído para cada aluno. Os questionários foram preenchidos individualmente. $\bigcirc$ pesquisador permaneceu na sala durante o preenchimento dos questionários, o que em média teve duração de 20 minutos, recolhendo-os quando todos os escolares tivessem terminado.

Para a análise estatística foi utilizado o Statistical Package for the Social Sciences (versão 18.0). Os hábitos posturais e comportamentais foram analisados separadamente para cada sexo e para cada grupo etário (Grupo I - I I e 12 anos, Grupo 2 - 13 e 14 anos e Grupo 3 - 15 e 16 anos), por meio de estatística descritiva. Foi utilizado também o teste Qui-quadrado para verificar a existência de associação entre os hábitos posturais e comportamentais e o sexo e grupo etário (THOMAS; NELSON, 2002; PESTANA; GAGEIRO, 2003) ( $\alpha=0,05)$.

\section{RESULTADOS}

Os resultados, em geral, demonstraram alta prevalência de hábitos posturais inadequados nas seguintes AVD 's: sentar para escrever, sentar em um banco, sentar para utilizar o computador e pegar objeto do chão. Em contrapartida, os resultados demonstraram também alta prevalência de hábitos posturais adequados nas AVD's dormir e transportar o material escolar, tanto no meio de transporte quanto no modo utilizado para este transporte. Além disso, os resultados demonstraram também que grande parte dos escolares permanece por mais de $4 \mathrm{~h}$ por dia na posição sentada em frente ao computador e à televisão, e que 49,3\% não dorme o tempo necessário recomendado pela literatura, de 8 a $9 \mathrm{~h}$ por noite (TABELA 2).

Quanto aos resultados referentes à associação entre hábitos posturais inadequados e comportamentais e sexo, na Tabela 2 pode-se perceber que: ( I ) a postura inadequada sentada em uma cadeira e/ou banco, (2) o hábito de ler e/ou estudar na cama, e (3) o meio e o modo de transporte do material escolar de forma inadequada estão associados significativamente ao sexo feminino, enquanto que tanto a postura inadequada para pegar objeto do chão quanto o tempo inadequado de sono por noite estão associados ao sexo masculino. 
Tabela 2. Prevalência de hábitos posturais e comportamentais em escolares do Ensino Fundamental de Teutônia, Rio Grande do Sul, Brasil, para ambos os sexos

\begin{tabular}{ccccc}
\hline Variáveis (N) & Total & Masculino & Feminino & $\chi^{\mathbf{2} \text { a }}$ \\
& $\mathrm{N}(\%)$ & $\mathrm{N}(\%)$ & $\mathrm{N}(\%)$ & \\
\hline
\end{tabular}

Tempo assistindo televisão por dia $(n=1349)$

$\begin{array}{lcccc}0 \text { a 3h } & 756(56) & 436(58,2) & 320(53,3) & \\ 4 \text { a 7h } & 463(34,3) & 243(32,4) & 220(36,7) & 0,193 \\ \geq 8 h & 130(9,6) & 70(9,3) & 60(10) & \end{array}$

Tempo utilizando o computador por dia $(n=1210)$

$\begin{array}{lllll}0 \text { a 3h } & 814(67,3) & 442(64,5) & 372(70,9) & \\ 4 \text { a 5h } & 249(20,6) & 153(22,3) & 96(18,3) & 0,067 \\ \geq 6 h & 147(12,1) & 90(13,1) & 57(10,9) & \end{array}$

Tempo de sono por noite $(n=1337)$

$\begin{array}{ccccc}0 \text { a 7h } & 447(33,4) & 269(36,2) & 178(30) & \\ 8 \text { a 9h (Adequado) } & 677(50,7) & 357(48) & 320(54) & 0,048^{c} \\ \geq 10 h & 213(15,9) & 118(15,9) & 95(16) & \end{array}$

Ler e/ou estudar na cama $(n=1597)$

$\begin{array}{ccccc}\text { Não } & 316(19,8) & 246(28,7) & 70(9,4) & \\ \text { Às vezes } & 792(49,6) & 428(50) & 364(49,3) & 0,00 I^{c} \\ \text { Sim } & 488(30,6) & 182(21,3) & 306(41,3) & \end{array}$

Postura para dormir $(n=\mid 449)$

Decúbito dorsal (Adequada)

Decúbito lateral (Adequada)

Decúbito ventral (Inadequada)

Postura sentada para escrever $(n=\mid 552)$

$\begin{array}{lllll}\text { Adequada } & 234(15,1) & 120(14,5) & 114(15,7) & \\ \text { Inadequada } & 1318(84,9) & 706(85,5) & 612(84,3) & 0,519\end{array}$

Postura sentada em uma cadeira e/ou banco

$$
(n=154 \mid)
$$

Adequada

$204(13,2) \quad 139(17) \quad 65(9)$

Inadequada

$1337(86,8) \quad 677(83) \quad 660(91) \quad 0,001^{c}$

Postura sentada para utilizar o computador $(n=1545)$ 


\begin{tabular}{|c|c|c|c|c|}
\hline Variáveis (N) & $\begin{array}{l}\text { Total } \\
\mathrm{N}(\%)\end{array}$ & $\begin{array}{l}\text { Masculino } \\
\mathrm{N}(\%)\end{array}$ & $\begin{array}{l}\text { Feminino } \\
\mathrm{N}(\%)\end{array}$ & $\chi^{2 \mathrm{a}}$ \\
\hline Adequada & $332(21,5)$ & $175(21,2)$ & $157(2 \mid, 9)$ & \\
\hline Inadequada & $1213(78,5)$ & $652(78,8)$ & $561(78,1)$ & 0,736 \\
\hline \multicolumn{5}{|l|}{ Postura para pegar objeto do chão $(n=\mid 476)$} \\
\hline Adequada & $124(8,4)$ & $54(6,8)$ & $70(10,3)$ & \\
\hline Inadequada & |352(9|,6) & $74 I(93,2)$ & $6 \mid 1(89,7)$ & $0,016^{c}$ \\
\hline \multicolumn{5}{|l|}{$\begin{array}{l}\text { Meio de transporte do material escolar } \\
\qquad(\mathrm{n}=1596)\end{array}$} \\
\hline Mochila escolar de duas alças & $1473(92,3)$ & $802(93,8)$ & $67 \mid(90,6)$ & \\
\hline $\begin{array}{l}\text { Outro meio de transporte (pasta, bolsa } \\
\text { outros) }\end{array}$ & $123(7,7)$ & $53(6,2)$ & $70(9,4)$ & $0,015^{c}$ \\
\hline \multicolumn{5}{|l|}{$\begin{array}{l}\text { Modo de transporte da mochila escolar } \\
\qquad(n=\mid 457)^{b}\end{array}$} \\
\hline $\begin{array}{c}\text { Adequado (alças simétricas sobre os } \\
\text { ombros) }\end{array}$ & $|29|(88,6)$ & $733(92,4)$ & $558(84)$ & \\
\hline Inadequado (modo não simétrico) & $166(\mid 1,4)$ & $60(7,6)$ & $106(16)$ & $0,00 I^{c}$ \\
\hline
\end{tabular}

\footnotetext{
a Teste Qui-quadrado.

${ }^{b}$ Escolares que utilizaram a mochila escolar de duas alças como meio de transporte do material escolar.

c Associação significativa $(p<0,05)$
}

A Tabela 3 apresenta os resultados da prevalência de hábitos comportamentais e posturais para cada um dos três grupos etários. Quanto à associação entre hábitos posturais inadequados e os grupos etários, pode-se observar que com o avanço da idade ocorre um aumento significativo da prevalência de hábitos posturais inadequados na postura sentada (para escrever, em uma cadeira e/ou banco e para utilizar o computador) e no modo de transporte do material escolar. Além disto, com o avanço da idade percebe-se uma diminuição tanto do tempo adequado de sono diário bem quanto do hábito de ler e/ou estudar na cama (TABELA 3)

Tabela 3. Prevalência de hábitos posturais e comportamentais em escolares do Ensino Fundamental de Teutônia, Rio Grande do Sul, Brasil, para os grupos etários ( I - 12 anos; I3- 14 anos; I5- 16 anos)

$\begin{array}{ccccc} & \text { II e } 12 \text { anos } & 13 \text { e } 14 \text { anos } & 15 \text { e } 16 & \text { anos } \\ N(\%) & N(\%) & N(\%) & \chi^{2} \text { a } \\ & N(N) & & \end{array}$

Tempo assistindo televisão por dia $(n=1349)$

\begin{tabular}{ccccc} 
0a 3h & $269(54,9)$ & $240(53,2)$ & $147(66,8)$ & \\
& & & & 0,010 \\
4 a $7 \mathrm{~h}$ & $169(34,5)$ & $237(37,1)$ & $57(25,9)$ & $c$ \\
$\geq 8 h$ & $52(10,6)$ & $62(9,7)$ & $16(7,3)$ & \\
\hline
\end{tabular}




\begin{tabular}{|c|c|c|c|c|}
\hline Variáveis (N) & $\begin{array}{c}\text { I I e } 12 \text { anos } \\
\text { N(\%) }\end{array}$ & $\begin{array}{c}13 \text { e } 14 \text { anos } \\
\text { N(\%) }\end{array}$ & $\begin{array}{c}15 \text { e } 16 \\
\text { anos } \\
\mathrm{N}(\%)\end{array}$ & $\chi^{2 \mathrm{a}}$ \\
\hline \multicolumn{5}{|c|}{ Tempo utilizando o computador por dia $(n=|2| 0)$} \\
\hline 0 a $3 h$ & $327(73,8)$ & $363(62,7)$ & $124(66)$ & \\
\hline & & & & 0,004 \\
\hline 4 a $5 h$ & $69(15,6)$ & $|4|(24,4)$ & $39(20,7)$ & c \\
\hline$\geq 6 h$ & $47(10,6)$ & $75(13)$ & $25(13,3)$ & \\
\hline \multicolumn{5}{|l|}{ Tempo de sono por noite $(n=\mid 337)$} \\
\hline $0 \mathrm{a} 7 \mathrm{~h}$ & $129(27,3)$ & $22 I(34,5)$ & $97(43,3)$ & \\
\hline & & & & 0,001 \\
\hline 8 a 9h (Adequado) & $254(53,8)$ & $323(50,4)$ & $100(44,6)$ & \\
\hline$\geq 10 \mathrm{~h}$ & $89(18,3)$ & $97(15,1)$ & $27(\mid 2,1)$ & \\
\hline \multicolumn{5}{|l|}{ Ler e/ou estudar na cama $(n=\mid 597)$} \\
\hline Não & $93(16,1)$ & $140(18,6)$ & $83(31,1)$ & \\
\hline & & & & 0,001 \\
\hline Às vezes & $3 \mid 4(54,2)$ & $358(47,7)$ & $120(44,9)$ & c \\
\hline Sim & $172(29,7)$ & $253(33,7)$ & $63(23,6)$ & \\
\hline \multicolumn{5}{|l|}{ Postura para dormir $(n=\mid 449)$} \\
\hline Decúbito dorsal (Adequada) & $43(8,1)$ & $57(8,4)$ & $15(6,3)$ & \\
\hline Decúbito lateral (Adequada) & $355(67,1)$ & $429(62,9)$ & 150(63) & 0,335 \\
\hline Decúbito ventral (Inadequada) & $131(24,8)$ & $196(28,7)$ & $73(30,7)$ & \\
\hline \multicolumn{5}{|l|}{ Postura sentada para escrever $(n=1552)$} \\
\hline Adequada & $113(19,9)$ & $92(12,7)$ & $29(11,2)$ & 0,001 \\
\hline Inadequada & $454(80,1)$ & $635(87,3)$ & $229(88,8)$ & c \\
\hline \multicolumn{5}{|c|}{ Postura sentada em uma cadeira e/ou banco $(n=|54|)$} \\
\hline Adequada & $94(16,7)$ & $82(11,4)$ & $28(\mid 1)$ & 0,010 \\
\hline Inadequada & $470(83,3)$ & $640(88,6)$ & $227(89)$ & c \\
\hline \multicolumn{5}{|c|}{ Postura sentada para utilizar o computador $(n=1545)$} \\
\hline Adequada & $\mid 45(25,8)$ & $133(18,2)$ & $54(21,6)$ & 0,004 \\
\hline Inadequada & $4 \mid 8(74,2)$ & $599(81,8)$ & $196(78,4)$ & c \\
\hline \multicolumn{5}{|l|}{ Postura para pegar objeto do chão $(n=\mid 476)$} \\
\hline Adequada & $4 I(7,6)$ & $57(8,2)$ & $26(10,8)$ & \\
\hline Inadequada & $500(92,4)$ & $637(91,8)$ & $215(89,2)$ & 0,318 \\
\hline \multicolumn{5}{|c|}{ Meio de transporte do material escolar $(n=1596)$} \\
\hline Mochila escolar de duas alças & $534(92,2)$ & $704(93,7)$ & $253(88,3)$ & \\
\hline Outro meio de transporte (pasta, bolsa & & & & 0,018 \\
\hline e outros) & $45(7,8)$ & $47(6,3)$ & $31(11,7)$ & c \\
\hline \multicolumn{5}{|l|}{ Modo de transporte da mochila escolar $(n=\mid 457)^{b}$} \\
\hline $\begin{array}{c}\text { Adequado (alças simétricas sobre os } \\
\text { ombros) }\end{array}$ & $466(88,3)$ & $620(88,4)$ & $205(89,9)$ & \\
\hline Inadequado (modo não simétrico) & $62(11,7)$ & $81(11,6)$ & $23(10,1)$ & 0,792 \\
\hline
\end{tabular}




\section{DISCUSSÃO}

Este estudo teve como objetivo verificar a prevalência de hábitos posturais inadequados em escolares do Ensino Fundamental da cidade de Teutônia, a partir de um estudo de base populacional. Os resultados sugerem que: (I) os escolares apresentam alta prevalência de hábitos inadequados nas posturas sentar para escrever, sentar em um banco, sentar para utilizar o computador e pegar objeto do chão; (2) o sexo feminino está associado com a postura inadequada sentada em uma cadeira e/ou banco, hábito de ler e/ou estudar na cama, meio e modo de transporte do material escolar; (3) o sexo masculino está associado à postura inadequada para pegar um objeto do chão e o tempo inadequado de sono por noite; e (4) com o avanço da idade ocorre um aumento da postura inadequada sentada, seja em uma cadeira/banco, para escrever ou para utilizar o computador, e percebe-se uma diminuição do tempo adequado de sono diário; (5) como aspecto positivo destaca-se que o avanço da idade está associado com a diminuição do hábito de ler e/ou estudar na cama.

Os resultados encontrados referentes aos hábitos posturais nas AVD's de sentar em um banco, sentar para escrever e sentar para utilizar o computador, demonstraram baixa prevalência de postura adequada, de 13,2\%, 15, 1\%, 21,5\% respectivamente (TABELA 2). Resultados semelhantes foram encontrados por Detsch et al. (2007), pois apenas 7,4\% e 27,5\% dos avaliados apresentaram hábitos posturais adequados ao assistir televisão e ao utilizar o computador, respectivamente. Estes resultados são ainda mais preocupantes se vistos conjuntamente com o tempo de permanência diário dedicado pelos escolares em atividades sedentárias. Do total de avaliados, 44\% permanecem sentados por mais de $4 \mathrm{~h}$ assistindo televisão e 26,2\% dos escolares utilizam o computador por mais de 4h diárias (TABELA 2). Esses resultados corroboram com Vitta et al. (20 I I) que, ao investigarem I236 escolares do Ensino Fundamental de Bauru, São Paulo, encontraram que 73\% dos escolares assistem televisão de 2 ou mais horas por dia, e 32,9\% utilizam o computador $2 \mathrm{~h}$ ou mais por dia. Resultados similares também foram encontrados por Martínez-Crespo et al. (2009), que ao pesquisarem os hábitos comportamentais de 887 jovens escolares da cidade de Sevilha, Espanha, encontraram que a maioria dos avaliados passava mais de $2 \mathrm{~h}$ diárias assistindo televisão. Com base em todos esses dados, e sabendo-se que os escolares em geral permanecem em média de 4 a 5 h diárias na posição sentada durante os períodos regulares de ensino, 5 vezes por semana (FREIRE; TEIXEIRA; SALES, 2008), especula-se que mais de 50\% dos escolares permanecem no mínimo 8h diárias em uma posição sentada, a qual caracteriza-se como uma atividade sedentária, sendo este um importante fator de risco para a ocorrência de alterações posturais (MARTíNEZ-CRESPO et al., 2009), 
entre outros problemas, como sobrepeso (TERRES et al., 2006; FONSECA et al., 1998), obesidade (PIMENTA; PALMA, 200 I) e problemas cardiovasculares (SILVA et al., 2005).

Do mesmo modo, os escolares que permanecem na posição sentada por longos períodos ao dia, estando grande parte deste tempo em postura inadequada (flexão anterior do tronco, falta de apoio lombar e falta de apoio do antebraço) estão predispostos a maiores índices de desconfortos gerais, tais como fadiga e formigamento em diferentes partes do corpo e, principalmente, a processos degenerativos nas estruturas osteoarticulares da coluna vertebral (CANDOTTI; NOLL; CRUZ, $2010 ;$ WOMERSLEY; MAY, 2006). Além disto, a grande quantidade de alunos em sala de aula e a necessidade de impor os limites levam os professores ao controle e rigidez no dia a dia escolar, fatos refletidos na exigência da manutenção da posição sentada dos escolares, acentuando os problemas relacionados à postura sentada e ao sedentarismo (FREIRE; TEIXEIRA; SALES, 2008). Embora os resultados (TABELA 2) demonstrem que o sexo feminino está associado com postura sentada inadequada, esta parece ser uma realidade comum a ambos os sexos, tendo em vista que mais de $75 \%$ dos escolares, de ambos os sexos, apresentam hábitos inadequados nas posturas sentar para escrever, sentar em um banco e sentar para utilizar o computador.

Não obstante, além da postura sentada, outras AVD's também são realizadas diariamente pelos escolares, como por exemplo, o ato de pegar objetos do chão, o qual, por sua vez, é uma tarefa realizada não somente no contexto da escola, mas também nas atividades domésticas e esportivas. No presente estudo, apesar do sexo masculino apresentar associação significativa com a postura inadequada ao pegar objetos do chão, o sexo feminino também apresenta alta prevalência de postura inadequada ao realizar a tarefa de pegar objetos do chão (TABELA 2). Assim como nas demais AVD's, a postura inadequada durante o ato de levantar objetos do chão pode provocar dor nas costas devido à sobrecarga na musculatura e nos ligamentos da região lombar. Além disso, em longo prazo, pode provocar sérios danos à estrutura do disco intervertebral (WILKE et al. , 1999; STRAKER, 2003). Uma possível explicação para esse resultado reside no fato de que a maioria das pessoas acredita conhecer a maneira adequada de erguer um objeto do solo, entretanto a maioria não a utiliza (OLIVER, 1999). Ao invés de realizar a tarefa de levantar um objeto do chão a partir da técnica de agachamento, ou seja, com flexão de joelhos e quadril, as pessoas geralmente optam por realizá-la com os joelhos estendidos e com flexão da coluna (STRAKER, 2003). Nesse sentido, especula-se que o motivo da não utilização da técnica adequada para levantar um objeto do chão pela maioria dos escolares esteja relacionado à tendência natural do ser humano em realizar 
as suas atividades em um menor gasto energético possível, que não é o caso do levantamento com agachamento, onde a fadiga é induzida mais precocemente (STRAKER, 2003).

Os resultados do presente estudo também evidenciaram que 21 , 3\% e 41,3\% dos meninos e meninas, respectivamente, possuem o hábito de realizar atividades de leitura e/ou estudo na cama. Esta atividade é desaconselhável visto que dificilmente o escolar a realizará em uma postura adequada. Este é um resultado interessante, pois demonstra características distintas entre os sexos, sendo que nesta AVD as meninas apresentam uma prevalência maior de hábito inadequado (TABELA 3). Além disso, os resultados demonstraram que o hábito de realizar atividades de leitura e/ ou estudo na cama tem diminuído com o avanço da idade, caracterizando-se como um aspecto positivo de comportamento dos escolares. Não obstante, o motivo da "perda" desse hábito inadequado não está completamente compreendido.

Ainda quanto ao avanço da idade, nota-se uma diminuição da quantidade diária de sono (TABELA 3), fato que coloca os escolares de Teutônia em situação de risco, pois tem sido referido, preliminarmente, que quantidades insuficientes de sono (PAANANEN et al., 20 I0), aliadas ao cansaço, dificuldades em adormecer, acordar no meio da noite, entre outros problemas (AUVINEN et al. , 20 I0), estão associadas às dores musculoesqueléticas.

No que diz respeito à alta prevalência de postura adequada ao transportar o material escolar, que é oposta às prevalências encontradas para os demais hábitos posturais, especula-se que esse resultado pode ser efeito dos programas preventivos realizados, nos últimos anos, especificamente para o ensinamento desse hábito (FERNANDES; CASAROTTO; JOÃO, 2008), assim como a grande ênfase dada pela mídia, em geral. Já para os demais hábitos posturais não tem sido verificado intervenções e/ou iniciativas com tamanha frequência e intensidade (NOLL; CANDOTTI; VIEIRA, 20I2; NOLL; CANDOTTI; VIEIRA, 20।3).

Investigações como as do presente estudo são relevantes visto que seus resultados possibilitam direcionar o trabalho educacional e preventivo. Neste prisma, conhecer os hábitos mais prejudiciais e atuar sobre a correção dos mesmos é uma ótima alternativa para minimizar ou prevenir hábitos inadequados no ambiente escolar (FREIRE; TEIXEIRA; SALES, 2008; NOLL et al. , 201 2C) e, por consequência, prevenir que padrões assumidos nesta etapa da vida tornem-se permanentes na fase adulta (NOLL; CANDOTTI; VIEIRA, 20I2; NOLL; CANDOTTI; VIEIRA, 20I3).

Por fim, a partir dos resultados do presente estudo, conclui-se que existe alta prevalência de hábitos posturais inadequados nas AVD's: (I) que envolvam a postura sentada, estando significativamente associados ao sexo feminino e ao avanço da idade e (2) que envolvam o ato de pegar objetos do chão, estando associado 
significativamente ao sexo masculino. Não obstante, existe também alta prevalência de hábitos posturais adequados nas AVD's de dormir e transportar o material escolar. Os resultados demonstraram também que os escolares permanecem por muito tempo em atividades sedentárias (posição sentada em frente ao computador e à televisão), e que a maioria não dorme o tempo necessário recomendado pela literatura, fato que se agrava à medida que a idade dos escolares aumenta.

\section{Prevalence of Inadequate Habits of Students from Elementary School of Teutônia City: a Population Study}

ABSTRACT: This study aimed to determine the prevalence of inadequate habits of Elementary students of the city of Teutônia, state of Rio Grande do Sul from a population study. The 1597 school participants completed the Back Pain and Body Posture Evaluation Instrument (BackPEl) self-administered questionnaire. The results showed a high prevalence of inappropriate postural habits in the following positions: sitting to write and to use the computer, sitting on a bench, and picking up objects from the ground. It was also found that students remain long time sitting in front of the computer and television, and that they do not sleep the required hours per day. Studies like this are important because their results provide direct educational work. KEYWORDS: Posture; Behavior; Adolescent Health; Epidemiology.

\section{Prevalencia de los hábitos inadecuados de los estudiantes de la} escuela primaria de la ciudad de Teutônia: un estudio de población

RESUMEN: Este estudio tuvo como objetivo determinar la prevalencia de hábitos posturales inadecuados de los estudiantes primarios de la ciudad de Teutônia, Estado de Rio Grande do Sul, a partir de un estudio de la población. El 1597 los participantes de la escuela completaron lo cuestionario auto-administrado Back Pain and Body Posture Evaluation Instrument (BackPEI). Los resultados mostraron alta prevalencia de malos hábitos posturales en las siguientes posiciones: sentarse a escribir y usar la computadora, sentarse em uno banco y recoger objetos del suelo. También se encontró que los estudiantes permanecen mucho tiempo sentados frente al computador y la televisión, y no duermen la cantidad de horas requeridas por día. Estudios como este son importantes debido a que sus resultados permiten dirigir el trabajo educativo. PALABRAS CLAVE: Postura; conducta; salud del adolecente; epidemiologia.

\section{REFERÊNCIAS}

ANDRADE, S. C.; ARAÚJO, A. G.; VILAR, M. J. Escola de coluna: revisão histórica e sua aplicação na lombalgia crônica. Revista Brasileira de Reumatologia, São Paulo, v. 45, n. 4, p. 224-228, ago. 2005.

APARICIO, Q. . et al. Influence of the kind daily school stage in the weight pupils rucksacks. Fisioterapia, Madrid, v. 27, n. I, p. 6-15, 2005. 
AUVINEN, J. P. et al. Is insufficient quantity and quality of sleep a risk factor for neck, shoulder and low back pain? a longitudinal study among adolescents. European Spine Journal, Heidelberg, v. 19, n. 4, p. 64I-649, apr. 2010.

BRACKLEY, H. M.; STEVENSON, J. M. Are children's backpack weight limits enough? a critical review of the relevant literature. Spine, Philadelphia, v. 29, p. 21 84-2190, oct. 2004.

BURTON, A. K. et al. The natural history of low back pain in adolescents. Spine, Philadelphia, v. 21 , p. 2323-2328, oct. 1996.

CANDOTTI, C. T. et al. Efeitos de um programa de educação postural para crianças e adolescentes após oito meses do seu término. Revista Paulista de Pediatria, São Paulo, v. 29, n. 4, p. 577-583, dez. 20। I.

CANDOTTI, C. T. et al. Escola postural: uma metodologia adaptada para crianças. Revista Arquivos em Movimento, Rio de Janeiro, v. 5, n. 2, p. 34-49, 2009.

CANDOTTI, C. T.; NOLL, M.; CRUZ, M. Prevalência de dor lombar e os desequilíbrios musculares em manicures. Revista Arquivos em Movimento, Rio de Janeiro, v. 6, n. I , p. I 25 140, 2010.

CANDOTTI, C. T.; ROHR, J.; NOLL, M. A Educação postural como conteúdo curricular da Educação Física no ensino fundamental ii nas escolas da cidade de Montenegro/RS. Movimento, Porto Alegre, v. 17, n. 3, p. 57-77, jul./set. 2011.

CANDOTTI, C. T.; ROTH, E.; NOLL, M. Assessment of weight and mode of transport of school material in highschool education. Revista Paulista de Pediatria, São Paulo, v. 30, n. I, p. $100-106,2012$.

DETSCH, C. et al. Prevalência de alterações posturais em escolares do ensino médio em uma cidade no Sul do Brasil. Revista Panamericana de Salud Publica, Washington,v. 2 I, n. 4, p. 23 I-238, abr. 2007.

DETSCH, C.; CANDOTTI, C.T. A incidência de desvios posturais em meninas de 6 a 17 anos da cidade de Novo Hamburgo. Movimento, Porto Alegre, v. 7, n. I 5, p. 43-56, 200 I.

FERNANDES, S.M.S.; CASAROTTO R.A.; JOÃO, S.M.A. Effects of educational sessions on school backpack use among elementary school students. Revista Brasileira de Fisioterapia, São Carlos, v. 6, p. 447-453, nov./dez. 2008.

FONSECA, V. M. et al. Fatores associados à obesidade em adolescentes. Revista de Saúde Pública, São Paulo, v. 32, n. 6, p. 54l-559, dez. 1998.

FREIRE, I. A.; TEIXEIRA, T. G.; SALES, C. R. Hábitos posturais: diagnóstico a partir de fotografias. Conexões, Campinas, v. 6, n. 2, p. 28-41, 2008.

FURTADO, R. et al. Validation of the Brazilian-Portuguese version of the gesture behavior test for patients with non-specific chronic low back pain. Clinics, São Paulo, v. 64, n. 2, p. 83-90, feb. 2009. 
LIMON, S.; VALINSKY, L. J.; SHALOM, Y. B. Risk factors for low back pain in the elementary school environment. Spine, Philadelphia, v. 29, n. 6, p. 697-702, mar. 2004.

LIS, A. M. et al. Association between sitting and occupational LBP. European Spine Journal, Heidelberg, v. 16, n. 2, p. 283-289, feb. 2007.

MARTELLI, R. C.; TRAEBERT, J. Estudo descritivo das alterações posturais de coluna vertebral em escolares de 10 a 16 anos de idade. Revista Brasileira de Epidemiologia, São Paulo, v. 9, n. I, p. 87-93, mar. 2006.

MARTINEZ-CRESPO, G. et al. Dolor de espalda em adolescentes: prevalência y fatores associados. Rehabilitacion, Madrid, v. 43, n. 2, p.72-80, 2009.

NOLL, M.; CANDOTTI, C. T.; VIEIRA, A. Escola postural: revisão sistemática dos programas desenvolvidos para escolares no brasil. Movimento, Porto Alegre, v. I8, n. 4, p. 265-29I, out./dez. 2012.

NOLL, M.; CANDOTTI, C. T.; VIEIRA, A. Instrumentos de avaliação da postura dinâmica: aplicabilidade ao ambiente escolar. Fisioterapia em Movimento, Curitiba, v. 26, n. I, p. 203217 , jan./mar. 2013.

NOLL, M. et al. Prevalência de dor nas costas e fatores associados em escolares do ensino fundamental do município de Teutônia, Rio Grande do Sul. Revista Brasileira de Saúde Materno Infantil, Recife, v. 12, n. 4, p. 395-402, out./dez. 2012 a.

NOLL, M. et al. Back Pain and body posture evaluation instrument (backpei): development, content validation and reproducibility. International Journal of Public Health, Basel, 20 I 2b. (In press).

NOLL, M. et al. Alterações posturais em escolares do ensino fundamental de uma escola de Teutônia/RS. Revista Brasileira de Ciência e Movimento, São Paulo, v. 20, n. 2, p. 32-42, abr./jun. $2012 \mathrm{c}$.

OLIVER, J. Back care: an illustrated guide. São Paulo: Manole, 1999.

PAANANEN, M. V. et al. Risk factors for persistence of multiple musculoskeletal pains in adolescence: A 2-year follow-up study. European Journal of Pain, London, v. I 4, n. I 0, p. 1026 1032, nov. 2010.

PENHA, P. J. et al. Qualitative postural analysis among boys and girls of seven to ten years of age. Revista Brasileira de Fisioterapia, São Carlos, v. I2, n.5, p. 386-39I, sept./oct. 2008.

PESTANA, M. H.; GAGEIRO, J. N. Análise de dados para ciências sociais: a complementaridade do SPSS. Lisboa: Edições Silabo, 2003.

PIMENTA, A. P.; PALMA, A. Epidemiological profile of obesity in children: relationship between television, physical activity and obesity. Revista Brasileira de Ciência e Movimento, São Paulo, v. 9 , n. 4, p. 19-24, oct. 2001 . 
ROBBINS, M.; JOHNSON, I. P.; CUNLIFFE, C. Encouraging good posture in school children using computers. Clinical Chiropractic, Netherlands, v. 12, p. 35-44, mar. 2009.

ROSA NETO, F. Avaliação postural em escolares de la à 4a série do l o grau. Revista Brasileira de Ciência e Movimento, São Paulo, v. 5, n. 2, p. 7-1।, mar. I991.

SANTOS, C. I. et al. Ocorrência de desvios posturais em escolares do ensino público fundamental de Jaguariúna, São Paulo. Revista Paulista de Pediatria, São Paulo, v. 27, n. I, p.74-80, mar. 2009.

STRAKER, L. Evidence to support using squat, semi-squat and stoop techniques to lift low-lying objects. International Journal of Industrial Ergonomics, New York, v. 3 I , n. 3, p. I 49- I60, mar. 2003.

SEAH, S. H. H. et al. An exploration of familial association $s$ in spinal posture defined using a clinical grouping method. Manual Therapy, Edinburgh, v. I6, p. 50 I-509, oct. 20 II.

SIIVOLA, S. et al. Predictive factors for neck and shoulder pain: a longitudinal study in young adults. Spine, Philadelphia, v. 29, n. I5, p. 1662-1669, ago. 2004.

SILVA, M. A. M. et al. Prevalence of cardiovascular risk factors in child and adolescent students in the city of maceió. Arquivos Brasileiros de Cardiologia, São Paulo, v. 84, n. 5, p. 387-92, may 2005.

SMITH, A.; O'SULLIVAN, P.; STRAKER, L. Classification of sagittal thoraco-lumbo-pelvic alignment of the adolescent spine in standing and its relationship to low back pain. Spine, Philadelphia, v. 33, n. 19, p. 2101-2117, sept. 2008.

TERRES, G. N. et al. Prevalence and factors associated to overweight and obesity in adolescents. Revista de Saúde Pública, São Paulo, v. 40, n. 4, p. 627-633, aug. 2006.

THOMAS, J.; NELSON, J. Métodos de pesquisa em atividade fisica. Porto Alegre: Artmed, 2002.

VICKERY, S.; MOFFAT, M. Manual de manutenção e reeducação postural. Porto Alegre: Artmed, 2002.

VITTA, A. et al. Prevalence of lower back pain and associated factors in students. Cadernos de Saúde Pública, Rio de Janeiro, v. 27, n. 8, p. I520-1528, aug. 201 I.

WILKE, J. et al. New in vivo measurements of pressures in the intervertebral disc in daily life. Spine, Philadelphia, v. 24, n. 8, p.755-762, apr. 1999.

WHITTFIELD, J.; LEGG, S. J.; HEDDERLY, D. I. Schoolbag weight and musculoskeletal symptoms in New Zealand secondary schools. Applied Ergonomics, Oxford, v. 36, p. $193-$ 198, mar. 2005.

WOMERSLEY, L.; MAY, S. Sitting posture of subjects with postural backache. Journal of Manipulative and Physiological Therapeutics, Arlington, v. 20, n. 3, p. 213-218, mar. 2006. 
Recebido em: 12 jan. 2012 Aprovado em: 10 dez. 2012

Endereço para Correspondência:

Matias Noll

Rua Guilherme Brust, 742

Bairro Languiru - Teutônia - RS

CEP: 95890-000 
Instrumento de Avaliação da Postura Corporal e Dor nas costas (BackPEI)

\section{Prezada aluna !!!}

Por favor responda com cuidado e atenção. Marque apenas uma alternativa para cada pergunta.

Caso tenha alguma dúvida, chame o responsável pela aplicação do questionário.

Nome: $\_$Data de nascimento: $\_$_
Peso: $\_$kg Estatura: $\_$cm $\quad$ Sexo: $\square$ Masculino $\square$ Feminino $\quad$ Ano: $\square 5^{\circ} \square 6^{\circ} \square 7^{\circ} \square 8^{\circ} \square 9^{\circ}$
Nome da Escola:
Nome do Pai (ou responsável):
Nome da Mãe (ou responsável):

1. Você pratica algum exercício físico ou esporte regularmente (na escola ou fora dela)?

$$
\square \text { sim, qual? — } \square \text { não (pule para a questão 4) }
$$

2. Quantos dias você pratica este exercício/esporte por semana?
$\square$ de 1 a 2 dias por semana
$\square$ ou mais dias por semana
$\square$ de 3 a 4 dias por semana
$\square$ não sei responder, depende da semana

3. Você pratica este exercício físico ou esporte de maneira competitiva (participa de competições)?

$$
\square \operatorname{sim} \quad \square \text { não }
$$

4. Quantas horas por dia você permanece sentado assistindo televisão?
$\square$ de 0 a 1 hora por dia
$\square$ de 6 a 7 horas por dia
$\square$ de 2 a 3 horas por dia
$\square 8$ horas ou mais por dia
$\square$ de 4 a 5 horas por dia
$\square$ não sei responder, depende do dia

5. Quantas horas por dia você permanece sentado utilizando o computador?
$\square$ de 0 a 1 hora por dia
$\square$ de 4 a 5 horas por dia
$\square$ não sei responder, depende do dia
$\square$ de 2 a 3 horas por dia
$\square 6$ horas ou mais por dia

6. Você costuma ler e/ou estudar na cama?
$\square \operatorname{sim}$
$\square$ não
$\square$ às vezes

7. Qual a sua posição preferida para dormir?
$\square$ de lado
$\square$ de costas (barriga para cima)
$\square$ de bruços (barriga para baixo)
$\square$ não sei responder, depende do dia

8. Quantas horas você dorme por noite?
$\square$ de 0 a 6 horas por dia
$\square$ de 8 a 9 horas por dia
$\square 7$ horas por dia
$\square 10$ horas ou mais por dia

9. Como você costuma sentar na escola para escrever à mesa ?

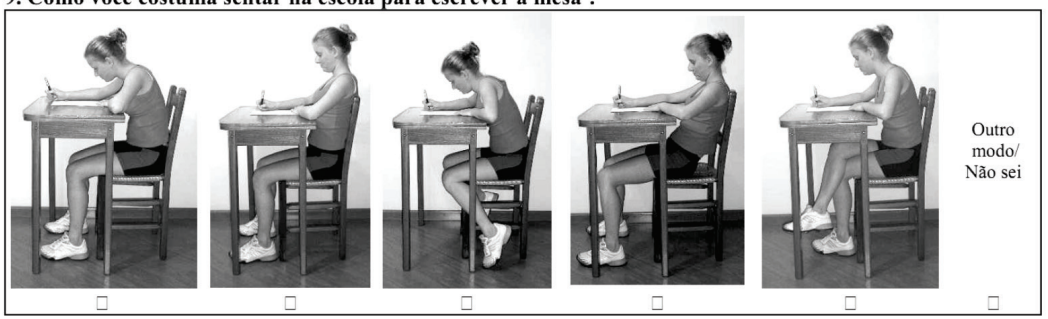


10. Como você costuma sentar em uma cadeira ou em um banco para conversar com os amigos?

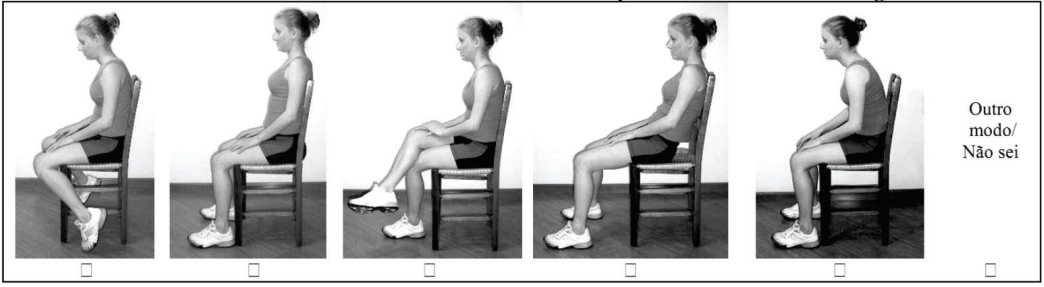

11. Como você costuma sentar para utilizar o computador?

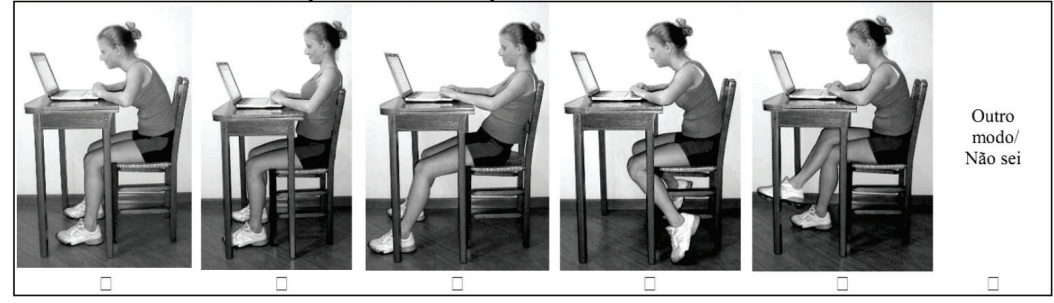

12. Como você costuma pegar objeto do chão?

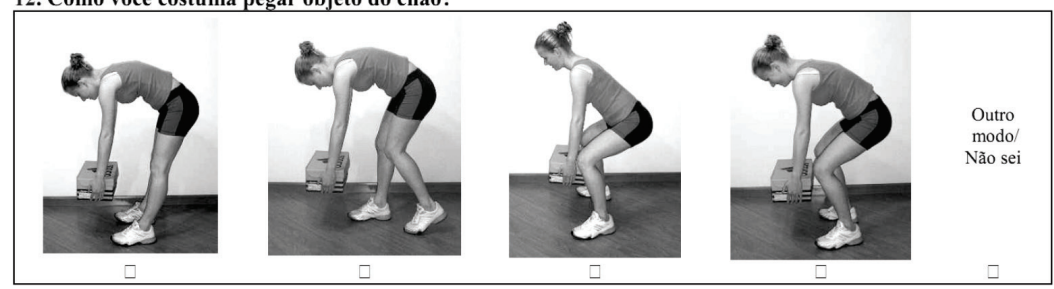

13. O que você utiliza para carregar o material escolar? Marque uma das opções abaixo.

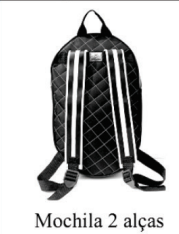

(a)

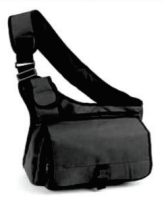

Mochila 1 alça

(b)

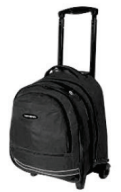

Mochila de rodas

(c)

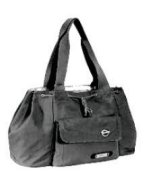

Bolsa

(d)

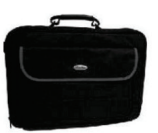

Outro

Pasta

(e)
Outro

(f)

Se você marcou a alternativa (a) (2 alças) responda a questão 14. Caso contrário, pule para a questão 15 . 
14. Como você leva sua mochila escolar?

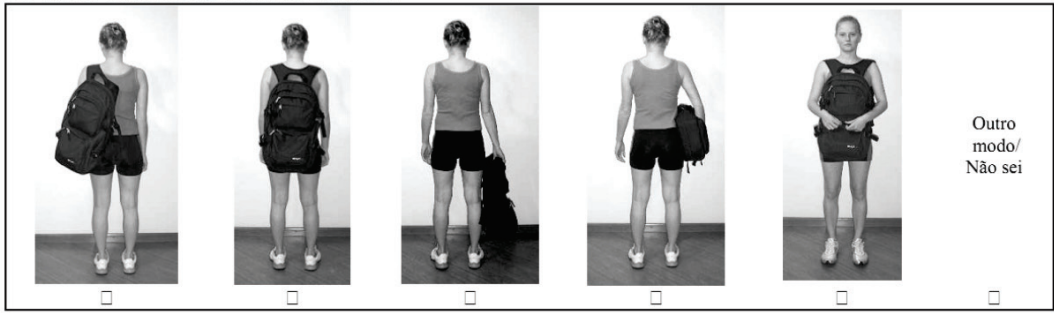

15. Até que série sua mãe (ou sua responsável do sexo feminino) estudou?
$\square$ não freqüentou a escola
$\square$ nível fundamental ( $1^{\mathrm{a}}$ a $8^{\mathrm{a}}$ série)
$\square$ nível superior (faculdade)
$\square$ nível médio ( $1^{\mathrm{a}}$ ano ao $3^{\circ}$ ano)
$\square$ não sei
$\square$ não tenho responsável do sexo feminino

16. Até que série seu pai (ou seu responsável do sexo masculino) estudou?
$\square$ não freqüentou a escola
$\square$ nível fundamental $\left(1^{\mathrm{a}}\right.$ a $8^{\mathrm{a}}$ série $)$
$\square$ nível superior (faculdade)
$\square$ nível médio ( $1^{\mathrm{a}}$ ano ao $3^{\circ}$ ano)
$\square$ não sei
$\square$ não tenho responsável do sexo masculino

17. Algum de seus pais (ou responsáveis) apresenta dor nas costas?
$\square$ Não sei responder
$\sqsubset \mathrm{Sim}$, quem?
¿ Não

18. Você sente ou já sentiu dor nas costas nos últimos 3 meses ?

$\square \operatorname{sim}$ (continue o questionário) $\square$ não (você encerrou o questionário, muito obrigado) $\square$ não sei responder

19. Esta dor nas costas ocorre ou ocorreu com que frequência?
$\square$ foi apenas uma vez
$\square$ uma vez por mês
$\square$ de duas a três vezes por semana
$\square$ uma vez por semana
$\square$ quatro vezes ou mais por semana
$\square$ não sei responder

20. Esta dor nas costas impede ou impediu de realizar atividades como: brincar, estudar, praticar esportes..
$\square \operatorname{sim}$
$\square$ não
$\square$ não sei responder

21. Na escala abaixo de 0 a 10, por favor, identifique a intensidade da dor nas costas nos últimos 3 meses (Faça um " $\mathrm{X}$ " sobre a linha abaixo de acordo com a intensidade da sua dor).

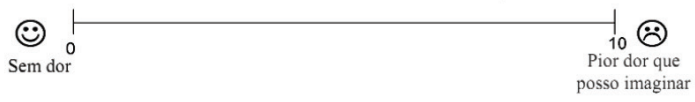

Muito obrigado pela atenção e colaboração. Tenha uma ótima semana !!! 


\section{Instrumento de Avaliação da Postura Corporal e Dor nas costas (BackPEI)}

\section{Prezado aluno !!!}

Por favor responda com cuidado e atenção. Marque apenas uma alternativa para cada pergunta.

Caso tenha alguma dúvida, chame o responsável pela aplicação do questionário.

Nome: $\_$Data de nascimento: $\_$Estatura: $\_$cm $\quad$ Sexo: $\square$ Masculino $\square$ Feminino $\quad$ Ano: $\square 5^{\circ} \square 6^{\circ} \square 7^{\circ} \square 8^{\circ} \square 9^{\circ}$
Peso: $\_$kg Município:
Nome da Escola: $\_$
Nome do Pai (ou responsável):
Nome da Mãe (ou responsável):

1. Você pratica algum exercício físico ou esporte regularmente (na escola ou fora dela)?

$$
\square \text { sim, qual? }
$$

$\square$ não (pule para a questão 4 )

2. Quantos dias você pratica este exercício/esporte por semana?
$\square$ de 1 a 2 dias por semana
$\square 5$ ou mais dias por semana
$\square$ de 3 a 4 dias por semana
$\square$ não sei responder, depende da semana

3. Você pratica este exercício físico ou esporte de maneira competitiva (participa de competições)?

$$
\square \operatorname{sim} \quad \square \text { não }
$$

4. Quantas horas por dia você permanece sentado assistindo televisão?
$\square$ de 0 a 1 hora por dia
$\square$ de 2 a 3 horas por dia
$\square$ de 6 a 7 horas por dia
$\square$ de 4 a 5 horas por dia
$\square 8$ horas ou mais por dia
$\square$ não sei responder, depende do dia

5. Quantas horas por dia você permanece sentado utilizando o computador?
$\square$ de 0 a 1 hora por dia
$\square$ de 4 a 5 horas por dia
$\square$ não sei responder, depende do dia
$\square$ de 2 a 3 horas por dia
$\square 6$ horas ou mais por dia

6. Você costuma ler e/ou estudar na cama?
$\square \operatorname{sim}$
$\square$ não
$\square$ às vezes

7. Qual a sua posição preferida para dormir?
$\square$ de lado
$\square$ de bruços (barriga para baixo)
$\square$ de costas (barriga para cima)
$\square$ não sei responder, depende do dia

8. Quantas horas você dorme por noite?
$\square$ de 0 a 6 horas por dia
$\square$ de 8 a 9 horas por dia
$\square 7$ horas por dia
$\square 10$ horas ou mais por dia

9. Como você costuma sentar na escola para escrever à mesa ?

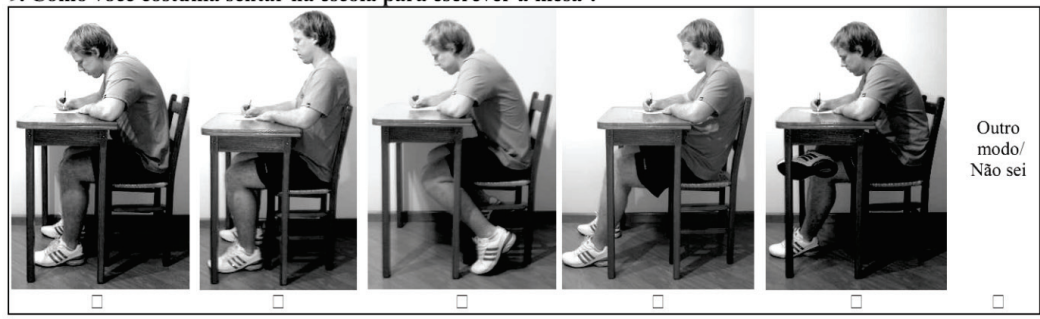


10. Como você costuma sentar em uma cadeira ou em um banco para conversar com os amigos?

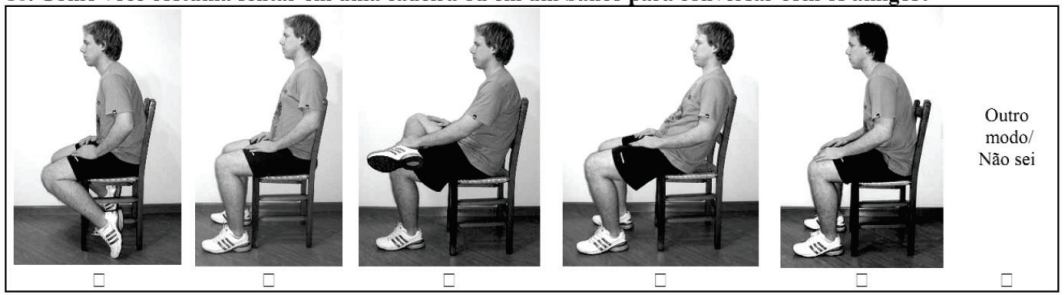

11. Como você costuma sentar para utilizar o computador?

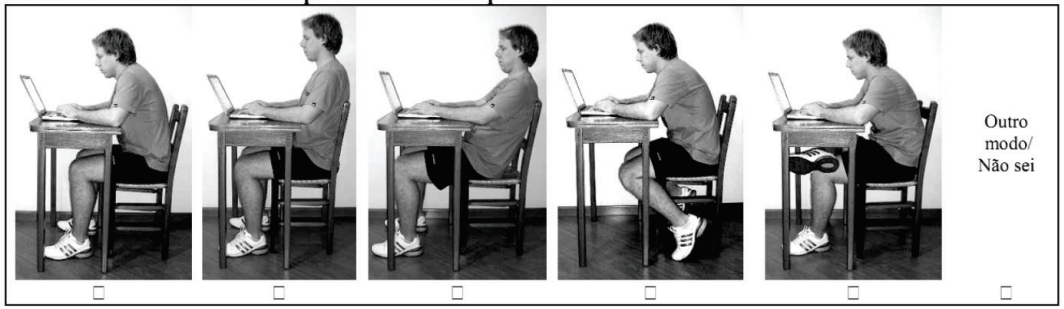

12. Como você costuma pegar objeto do chão?

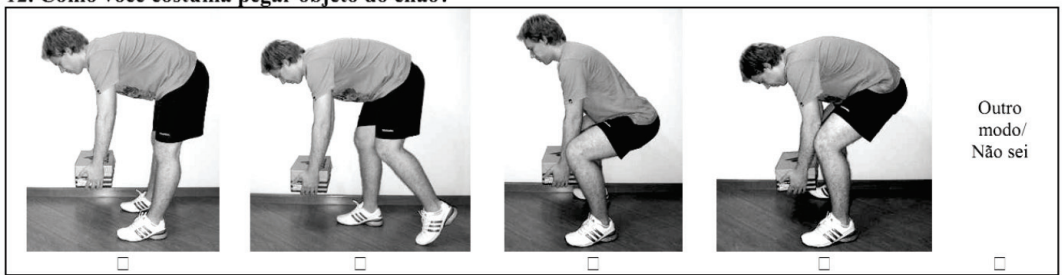

13. O que você utiliza para carregar o material escolar? Marque uma das opções abaixo.

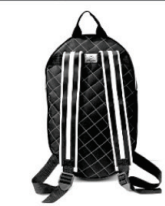

Mochila 2 alças

(a)

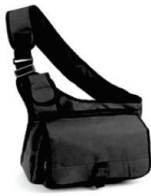

Mochila 1 alça

(b)

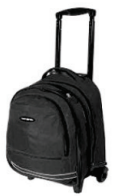

Mochila de rodas

(c)

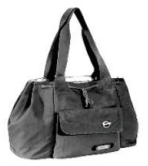

Bolsa

(d)

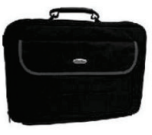

Pasta

(e )
Outro

(f)

Se você marcou a alternativa (a) ( 2 alças) responda a questão 14. Caso contrário, pule para a questão 15. 


\section{Como você leva sua mochila escolar?}

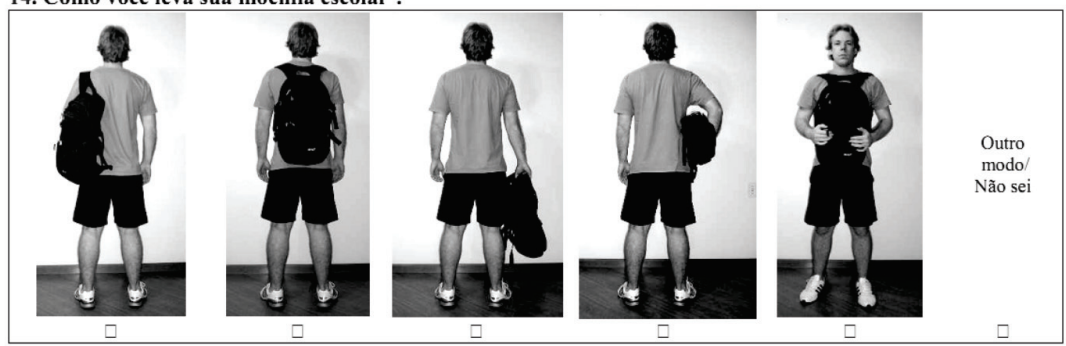

15. Até que série sua mãe (ou sua responsável do sexo feminino) estudou?
- não freqüentou a escola
$\square$ nível superior (faculdade)
- nivel fundamental ( $1^{\mathrm{a}}$ a $8^{\mathrm{a}}$ série)
$\square$ não sei
- nivel médio $\left(1^{\mathrm{a}}\right.$ ano ao $3^{\circ}$ ano $)$
๑ não tenho responsável do sexo feminino

16. Até que série seu pai (ou seu responsável do sexo masculino) estudou?
- não freqüentou a escola
$\square$ nivel fundamental $\left(1^{\mathrm{a}}\right.$ a $8^{\mathrm{a}}$ série $)$
$\square$ nível superior (faculdade)
- nivel médio ( $1^{\mathrm{a}}$ ano ao $3^{\circ}$ ano)
$\square$ não sei
$\square$ não tenho responsável do sexo masculino

17. Algum de seus pais (ou responsáveis) apresenta dor nas costas?
- Não sei responder
- Sim, quem?
$\square$ Não

18. Você sente ou já sentiu dor nas costas nos últimos 3 meses ?

$\square \operatorname{sim}$ (continue o questionário) $\square$ não (você encerrou o questionário, muito obrigado) $\square$ não sei responder

19. Esta dor nas costas ocorre ou ocorreu com que frequência?
च foi apenas uma vez
- uma vez por mês
$\beth$ de duas a três vezes por semana
$\square$ uma vez por semana
$\square$ quatro vezes ou mais por semana
$\square$ não sei responder

20. Esta dor nas costas impede ou impediu de realizar atividades como: brincar, estudar, praticar esportes...

$$
\square \operatorname{sim} \quad \square \text { não } \quad \square \text { não sei responder }
$$

21. Na escala abaixo de $\mathbf{0}$ a 10 , por favor, identifique a intensidade da dor nas costas nos últimos 3 meses (Faça um " $\mathrm{X}$ " sobre a linha abaixo de acordo com a intensidade da sua dor).

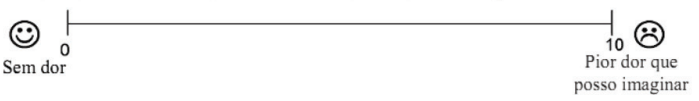

Muito obrigado pela atenção e colaboração.

Tenha uma ótima semana !!! 